\title{
Empyema and lung abscess: An unusual presentation of pulmonary actinomycosis
}

\author{
Matthew A. Robinson*1, Avinesh S. Bhar ${ }^{2}$ \\ ${ }^{1}$ Mercer University School of Medicine, Macon, United States \\ ${ }^{2}$ Navicent Health Medical Center of Central Georgia, Macon, United States
}

Received: March 27, 2017

DOI: $10.5430 /$ crim.v4n2p60
Accepted: April 27, 2017

URL: https://doi.org/10.5430/crim.v4n2p60

\begin{abstract}
Actinomyces spp. are an infrequent cause of pulmonary infections. Actinomyces spp. comprise part of the normal flora of the oral cavity and gastrointestinal tract. A. meyeri is especially known for pulmonary involvement and disseminated disease. The authors present a case of a polymicrobial pulmonary abscess containing A. meyeri. The patient was a 58-year-old man with a history of coronary artery disease, alcohol abuse and hypertension. He presented with progressive dyspnea and cough productive of grey-colored foul smelling sputum. Upon admission, a chest computed tomographic (CT) scan revealed a right-lower-lobe lung abscess, with an associated loculated empyema. The following day, the patient underwent a thoracostomy with chest tube placement. The chest tube drained several hundred milliliters of purulent pleural fluid, but a follow up chest CT showed little change in the size of the underlying empyema. A subsequent thoracotomy with decortication was performed, which evacuated $100 \mathrm{~mL}$ of thick purulent fluid. Fourteen days after admission, the patient was discharged on a 6- to 12-week course of intravenous penicillin G, followed by a 6-month course of oral penicillin V.
\end{abstract}

Key Words: Actinomyces meyeri, Pulmonary actinomycosis, Lung abscess, Empyema

\section{INTRODUCTION}

Actinomyces spp. are gram positive, branching, facultative anaerobes. They comprise part of the normal flora of the gastrointestinal tract, oral cavity, and female genitourinary tract. ${ }^{[1]}$ Cervicofacial infection is the most common manifestation of actinomycosis and typically presents as an oral lesion with draining sinus tracts that burrow to the skin or mucosal surfaces. ${ }^{[2]}$ On rare occasions, these organisms may be aspirated and can cause pneumonia or a pulmonary abscess. These infections are most common in those at increased risk for aspiration, including patients with neurological disease and those with chronic alcohol abuse. Pulmonary actinomycosis usually presents with fever, cough, and weight loss, and is often confused with tuberculosis or malignancy, due to the similarity in clinical presentation. ${ }^{[3,4]}$ Accurate diagnosis is imperative, because the presence of Actinomyces spp. alters the treatment course of lung abscesses.

\section{CASe PResentation}

A 58-year-old man with a medical history of hypertension and coronary artery disease had been treated as an outpatient for a right-lower-lobe pneumonia following a CXR ordered by his primary care physician. Two seven day courses of azithromycin and moxifloxacin (the first CXR ordered after the initial course of azithromycin) followed by a 14-day course of augmentin were prescribed over a period of about

\footnotetext{
*Correspondence: Matthew A. Robinson; Email: robinson.matthew340@ gmail.com; Address: Mercer University, School of Medicine, Macon, United States.
} 
two months with no symptomatic or radiological improvement. A repeat CXR following the course of augmentin revealed an associated effusion. Patient was then admitted to the hospital with a chest CT with contrast ordered after the third course of antibiotics failed to resolve his symptoms of cough and chest pain (see Figure 1). This CT revealed a right-lower-lobe cavitary lesion measuring $6.0 \mathrm{~cm} \times 4.3 \mathrm{~cm}$, with air fluid levels suggestive of an abscess but unable to exclude a neoplasm. A loculated pleural effusion was also seen measuring $11.0 \mathrm{~cm} \times 4.0 \mathrm{~cm}$ at the right lung base, suggestive of a hydropneumothorax or empyema, along with multiple enlarged paratracheal lymph nodes. These findings prompted his admission for further evaluation.

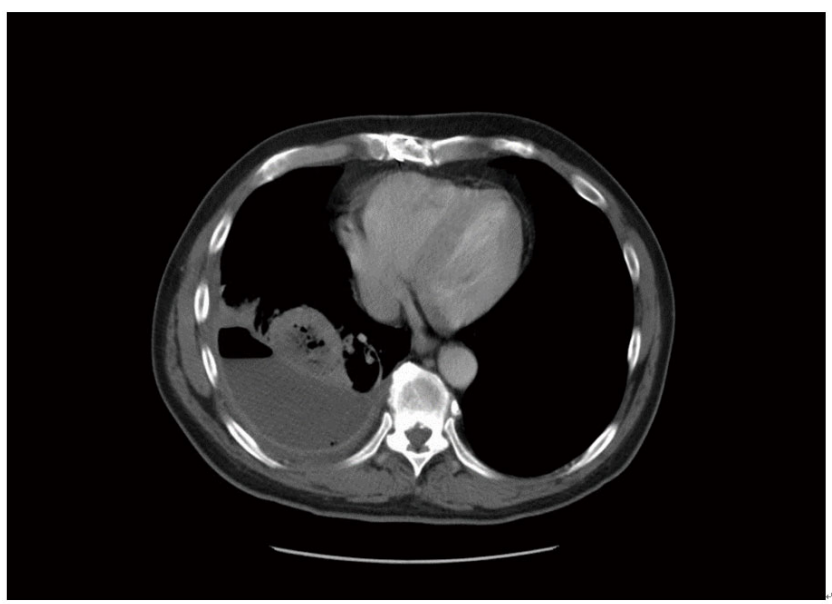

Figure 1. Non-contrast chest CT of right-lower-lobe abscess and empyema present on admission

On admission, the patient complained of dyspnea, cough productive of foul smelling sputum, and a sharp pleuritic pain at the right hypochondria. Social history included cigarette smoking, weekend binge drinking, and employment spraying glass lining into industrial chemical containers. On physical exam, the patient was afebrile and tachypneic. His dentition was poor, with a decaying molar on the lower right side. Lung auscultation revealed reduced breath sounds at the right lower lung base. Laboratory analysis demonstrated leukocytosis with a white blood cell (WBC) count of 16,000 cells $/ \mu 1$, and a hemoglobin value of $8.8 \mathrm{~g} / \mathrm{dl}$. Iron studies included a ferritin of $675.8 \mathrm{ng} / \mathrm{ml}$, a total iron binding capacity (TIBC) of $200 \mu \mathrm{g} / \mathrm{dl}$, and an iron saturation of $12 \%$. This pattern is consistent with inflammatory anemia.

The treatment plan included continuation of intravenous moxifloxacin at $400 \mathrm{mg}$ once a day, and a thoracostomy with chest tube placement was planned for the following day. The chest tube drained approximately $400 \mathrm{ml}$ of green, cloudy fluid over the subsequent 4 days, with the patient experiencing Published by Sciedu Press symptomatic improvement. Laboratory studies of the fluid revealed a WBC count of 570,000 cells $/ \mu 1$, with $44 \%$ polymorphonuclear leukocytes (PMNs), 49\% monocytes, and $7 \%$ lymphocytes. Gram staining of the fluid was significant for both gram positive cocci and gram positive rods. The high viscosity of the fluid prevented the assessment of lactate dehydrogenase (LDH) and total protein (dilution method was not attempted by the laboratory). Cultures of the fluid were also obtained to guide antibiotic treatment.

Six days after admission, a follow-up CT without contrast suggested a decreased but persistent empyema measuring $8.5 \mathrm{~cm} \times 2.3 \mathrm{~cm}$, and a marginally smaller right-lower-lobe cavitary lesion measuring $5.4 \mathrm{~cm} \times 4.4 \mathrm{~cm}$ (see Figure 2). At this time, the decision was made to perform a thoracotomy to thoroughly drain the abscess and empyema.

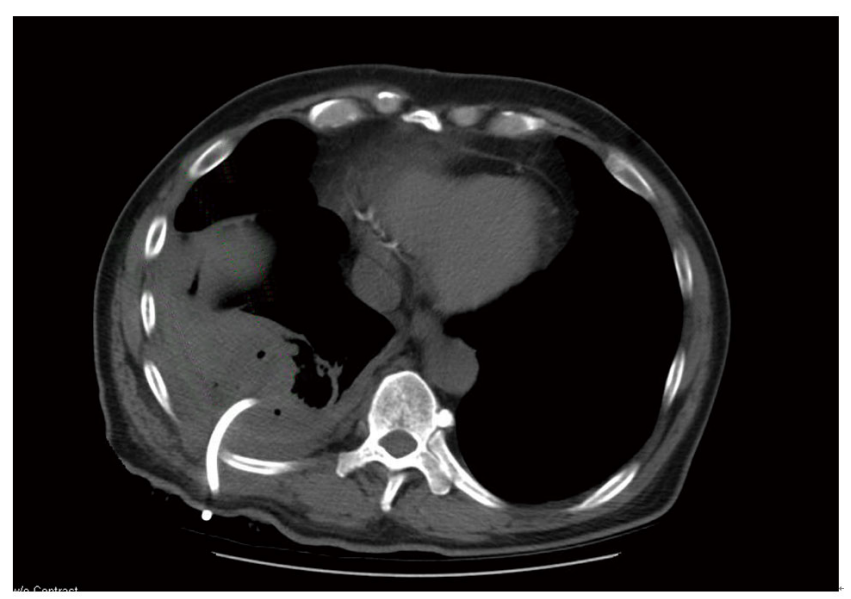

Figure 2. Unresolved right-lower-lobe abscess and empyema on hospital day 6

On hospital day 7 , the pleural fluid cultures obtained during the initial thoracostomy returned with heavy growth of Streptococcus constellatus and moderate growth of Actinomyces meyeri. Based on the sensitivities of the organisms, the patient's antibiotics were subsequently changed to intravenous ceftriaxone $2 \mathrm{gm}$ once a day.

On hospital day 8, a posterior muscle-sparing thoracotomy with decortication was performed to drain the abscess. Approximately $100 \mathrm{ml}$ of very viscous and purulent fluid was evacuated from the cavity. Two chest tubes were placed for continuous post-procedure drainage.

The patient had an uneventful surgical recovery. A peripherally inserted central catheter (PICC) line was placed for at-home antibiotic treatment. He was discharged on hospital day 14 on an antibiotic regimen of 6 to 12 weeks intravenous penicillin, followed by 6 months of oral penicillin. 


\section{Discussion}

Actinomyces spp. are a rare cause of pulmonary infections. The microbes are commensals in the oral cavity, gastrointestinal, and genitourinary tracts. ${ }^{[1]}$ Poor oral hygiene is a risk factor for pulmonary actinomycosis, particularly when paired with a condition that increases the risk of aspiration. Examples of these conditions include Parkinson's Disease, dementia, dysphagia, and alcohol abuse. The patient described was at increased risk, due to his poor oral hygiene and alcohol abuse.

Pulmonary actinomycosis can mimic a number of pulmonary conditions, including pneumonia, tuberculosis, and malignancy. Cough is the most commonly reported presentation, most often accompanied by sputum production and hemoptysis. Fever and chest pain are also common complaints. ${ }^{[5]}$ Because symptoms are generally nonspecific, the diagnosis is often delayed or missed entirely. ${ }^{[3,4]}$ Imaging techniques are nondiagnostic for actinomycosis, and misdiagnosis is common without microbiological or histological examination. As many as two thirds of patients in one study were misdiagnosed with malignancy, using ${ }^{18} \mathrm{~F}$-fluorodeoxyglucose positron-emission tomography (FDG-PET) in particular. ${ }^{[5]}$ Further confusing matters is the fact that Actinomyces spp. can sometimes colonize the necrotic tissues of lung neoplasms. ${ }^{[6]}$ Diagnosis requires isolation of Actinomyces spp. from a clinical specimen such as a bronchoscopic or surgical biopsy. However, culture is often falsely negative due to the organisms fastidious nature. ${ }^{[7]}$ The diagnosis can also be made by the observation of sulfur granules in the purulent debris from the site of infection. ${ }^{[6]}$

With pulmonary actinomycosis, accurate diagnosis is imperative, because the treatment course differs from pulmonary infections caused by other microbes. The preferred treatment for a typical polymicrobial pulmonary abscess consists of intravenous clindamycin $600 \mathrm{mg}$ every eight hours for 5 to 21 days, followed by 50 to $300 \mathrm{mg}$ oral clindamycin four times daily for 28 to 48 days. ${ }^{[8]}$ Lesions involving Actinomyces spp. are usually sensitive to several antibiotics, including penicillins and tetracyclines, but require longer treatment courses to attain resolution. This is possibly due to poor drug penetration as a result of sulfur granule aggregations of actinomyces. ${ }^{[5]}$ No guidelines for the duration of treatment have been published, but antibiotic courses of 6 to 12 months are typically necessary. ${ }^{[7]}$ Surgery is generally reserved for complications, such as abscess, empyema, or fistula formation. ${ }^{[9]}$

For properly treated pulmonary actinomycosis, prognosis is excellent. Antimicrobial therapy is highly effective, particularly with early diagnosis. ${ }^{[10]}$ Pulmonary physicians should consider this diagnosis if the patient has poor oral hygiene and an increased risk of aspiration, as early diagnosis and treatment may prevent future complications, such as abscess formation and empyema.

\section{CONFLicts OF InTEREST Disclosure}

The authors have declared no conflicts of interest.

\section{REFERENCES}

[1] Smego JRA, Foglia G. Actinomycosis. Clinical Infectious Diseases. 1998; 26(6): 1255-61. https ://doi.org/10.1086/516337

[2] Lerner PI. The lumpy jaw. Cervicofacial actinomycosis. Infectious Disease Clinics of North America. 1988 Mar 2: 203-20. PMid:3074108

[3] Kinnear W, Macfarlane J. A survey of thoracic actinomycosis. Respiratory Medicine. 1990; 84(1): 57-9. https ://doi.org/10.1016/ S0954-6111(08) 80095-9

[4] Schweigert M, Meyer C, Stadlhuber R, et al. Surgery for Inflammatory Tumor of the Lung Caused by Pulmonary Actinomycosis. The Thoracic and Cardiovascular Surgeon. 2012; 60(02): 156-60. PMid:21695671 https://doi.org/10.1055/s-0030-1271180

[5] Shi JH, Sun XF, Wang P, et al. A Retrospective Study of Pulmonary Actinomycosis in a Single Institution in China. Chinese
Medical Journal. 2015; 128(12): 1607. PMid:26063362 https: //doi.org/10.4103/0366-6999.158316

[6] Mabeza G, Macfarlane J. Pulmonary actinomycosis. European Respiratory Journal. 2003; 21(3): 545-51. https : //doi .org/10.118 3/09031936.03.00089103

[7] Wong VK, Turmezei TD, Weston VC. Actinomycosis. BMJ. 2011; 343: d6099. PMid:21990282

[8] Kuhajda I, Zarogoulidid K, Tsirgogianni K, et al. Lung abscessetiology, diagnostic and treatment options. Annals of Translational Medicine. 2015; 3(13): 183-91.

[9] Conant EF, Wechsler RJ. Actinomycosis and nocardiosis of the lung. Journal of Thoracic Imaging. 1992; 7(4): 75-84. https: //doi.org/10.1097/00005382-199209000-00010

[10] Kim SR, Jung LY, Oh IJ, et al. Pulmonary actinomycosis during the first decade of 21 st century: cases of 94 patients. BMC Infectious Diseases. 2013; 13(1). 